\title{
The influence of mass media on Italian web users during COVID-19: an infodemiological analysis.
}

\author{
Alessandro Rovetta ${ }^{1,2}$, Lucia Castaldo ${ }^{1,2}$ \\ ${ }^{1}$ Mensana srls, Research and Disclosure Division, Brescia, Italy \\ ${ }^{2}$ Redeev srl, Technological and Scientific Research, Napoli, Italy
}

\section{Corresponding author}

Alessandro Rovetta,

Mensana srls, Research and Disclosure Division,

Via Malta $12-25124$ Brescia, Italy.

Redeev Technological and Scientific Research,

Via San Pasquale a Chiaia 83, Naples, Italy.

Email: rovetta.mresearch@gmail.com

Phone: 39-3927112808

ORCID: 0000-0002-4634-279X

Web of Science Researcher ID: AAT-9063-2020

\section{Funding}

This research did not receive any specific grant from funding agencies in the public, commercial, or not-for-profit sectors.

\section{Author information}

Alessandro Rovetta designed the study, collected the data, performed the data analysis, and wrote the manuscript. Lucia Castaldo contributed to the collection and analysis of data.

\section{Copyright statement}

This work is copyrighted (Patamu deposit number: 151420). The certain date of the generation of the author's proof is certified with time stamps and its validity is guaranteed pursuant to the 910/2014 EU eIDAS regulation for Digital Trust services. 


\begin{abstract}
Alongside the COVID-19 pandemic, the world has had to face a growing infodemic, which has caused severe damage to economic and health systems and has often compromised the effectiveness of infection containment regulations. Although this has spread mainly through social media, there are numerous occasions in which the mass media have shared dangerous information, giving resonance to statements without a scientific basis. For these reasons, infoveillance and infodemiology methods are increasingly exploited to monitor online information traffic. However, these tools have also been used to make epidemiological predictions. In particular, the "Google Trends" tool by Google ${ }^{\mathrm{TM}}$ has often been adopted by the scientific community to carry out this type of analysis. In this regard, the purpose of this paper is to estimate the impact of Italian mass media on users' web searches in order to understand the role of the media in the infodemic and in the interest of Italian web users towards COVID-19. In particular, our results suggest that the Italian mass media have played a decisive role both in the spread of the infodemic and in addressing netizens' web interest, favoring the adoption of terms unsuitable for identifying the novel coronavirus (COVID- 19 disease). Therefore, we suggest greater caution and attention by the directors of news channels and newspapers and greater control of the latter by government dissemination agencies.
\end{abstract}




\section{Introduction}

The COVID-19 pandemic has put a strain on economies and health systems around the world [1, 2]. As of March 21, 2020, the death toll is around 2.71 million and the trend continues to grow [3]. However, in addition to the disease, the world has had to face a growing infodemic capable of causing damage of equal severity [4]. The role of social media (e.g. Facebook, Instagram and Twitter) and even traditional media (e.g. national newscasts and newspapers) in the spread of infodemic information has now been consolidated by a large literature [5-10]. Furthermore, there is strong evidence that these two communication sectors are not separate: in fact, mass media have been able to heavily influence the trends of users' web searches [10-12]. In such a vast and complex scenario, a quantitative estimate of this phenomenon is difficult to achieve as includes a wide range of factors. Nonetheless, the online behavior of users has often been monitored not only for mere infoveillance but also to make predictions at the epidemiological level, assess the psychological state and risk perception of web users or particular requests for medical assistance, and carry out analyzes of environmental sustainability [13-18]. Therefore, given this international crisis, the need for a deep understanding of the web media-user relationship is imperative. In this paper, we analyze the influence of the main Italian media on web users looking for causal correlations, cross-correlations, and relationships between the variables. Specifically, we investigate three aspects: i) the adoption of the most appropriate terms for the identification of SARS-CoV-2 and related COVID-19 by media and web users, assigning each term a specific value on the infodemic scale (I-scale, [10]), ii) any causal correlations and cross-correlations between article titles of the two most read newspapers in Italy "La Repubblica" and "Il Corriere della Sera" and web searches on Google, and iii) the contribution of Italian media in the spread of fake news. Data relating to media were collected on their official websites and YouTube channels, while web users' interest was evaluated through the infoveillance tool "Google Trends" by Google [19]. As highlighted by Cervellin et al. [20], Google Trends is heavily subjected to the media, which is why it is essential, during this international crisis, to dissect this relationship. Therefore, this paper aims to answer the following research questions: R1) how much have the Italian media influenced Italian web users in the adoption of terms to identify the novel coronavirus (COVID-19)? R2) how much have the Italian media influenced the web interest in the novel coronavirus (COVID-19)? R3) What was the role of the Italian mass media in the COVID19 infodemic? R4) Is Google Trends a reliable tool for epidemiological analysis based on symptomrelated web searches or does it reflect media interest in the topic? 


\section{Results}

\subsection{Media influence on Italian web users}

\section{Infodemic and scientific names: trends and correlations}

A substantial difference in the adoption of scientific names "2019-nCoV" and "novel coronavirus" against the more infodemic "coronavirus" was found at the beginning of the pandemic between the most read newspapers "La Repubblica", "Il Corriere della Sera", and others including "Il Sole 24 Ore", "Il Fatto Quotidiano", "Il Giornale" and "La Stampa", and sources like Google and PubMed (Table 1).

\begin{tabular}{rrrrr}
\hline & 2019-ncov & novel coronavirus & coronavirus & Chinese (corona)virus \\
\hline Infodemic Scale & 0 & 1 & 4 & 9 \\
\hline PubMed & $42.7 \%$ & $45.2 \%$ & $12.1 \%$ & $<0.1 \%$ \\
\hline Ministry of Health & $11.8 \%$ & $52.9 \%$ & $35.3 \%$ & $0.0 \%$ \\
\hline Google & $29.3 \%$ & $25.9 \%$ & $27.6 \%$ & $17.2 \%$ \\
\hline La Repubblica & $0.2 \%$ & $5.3 \%$ & $92.9 \%$ & $1.6 \%$ \\
\hline II Corriere d. Sera & $1.3 \%$ & $4.7 \%$ & $87.7 \%$ & $6.3 \%$ \\
\hline Other Newspapers* & $0.5 \%$ & $0.1 \%$ & $93.9 \%$ & $5.4 \%$ \\
\hline Rai (Google News) & $1.6 \%$ & $0.0 \%$ & $96.8 \%$ & $1.6 \%$ \\
\hline Mediaset (Google News) & $0.0 \%$ & $0.0 \%$ & $97.8 \%$ & $2.2 \%$
\end{tabular}

Table 1. Rate of adoption of COVID-19-related terms at the start of the pandemic (Jan 1 - Feb 13, 2020). *This item also includes "Il Sole 24 Ore", "Il Fatto Quotidiano", "Il Giornale", and "La Stampa".

Moreover, the streaming video portals "RaiPlay" and "MediasetPlay" by RAI and Mediaset (the main Italian television broadcasters) did not show any results for the item "2019-ncov" while produced several results for the queries "Chinese virus" and "Chinese coronavirus". These websites have also special sections entitled "coronavirus" [21, 22]. According to Google results, from 1 January to 13 February 2020, the same websites used the term "coronavirus" in 93.8\% of article titles against 6.3\% of "2019-ncov" and $0.0 \%$ "of novel coronavirus". In this context, the Italian web users immediately preferred the term "coronavirus" $\left(R S V_{a v}=14\right)$ despite all the others $(\forall R S V<1)$, showing values comparable to those of the most relevant newspapers and television broadcasters in Italy. Moreover, "Chinese virus" and "Chinese coronavirus" were the second most used queries during this phase $\left(R S V_{a v}=22 v s \forall R S V_{a v} \leq 1\right.$ excluding the query "coronavirus"), showing further similarities with the Italian media. Considering the keyword "coronavirus", the weekly-cumulative RSV showed a pronounced correlation with its weekly-cumulative use by the newspaper "La Repubblica" (Figure 1, next page). 
- coronavirus t.d.i. $\quad$ - covid t.d.i. $=$ coronavirus RSV $=$ covid RSV

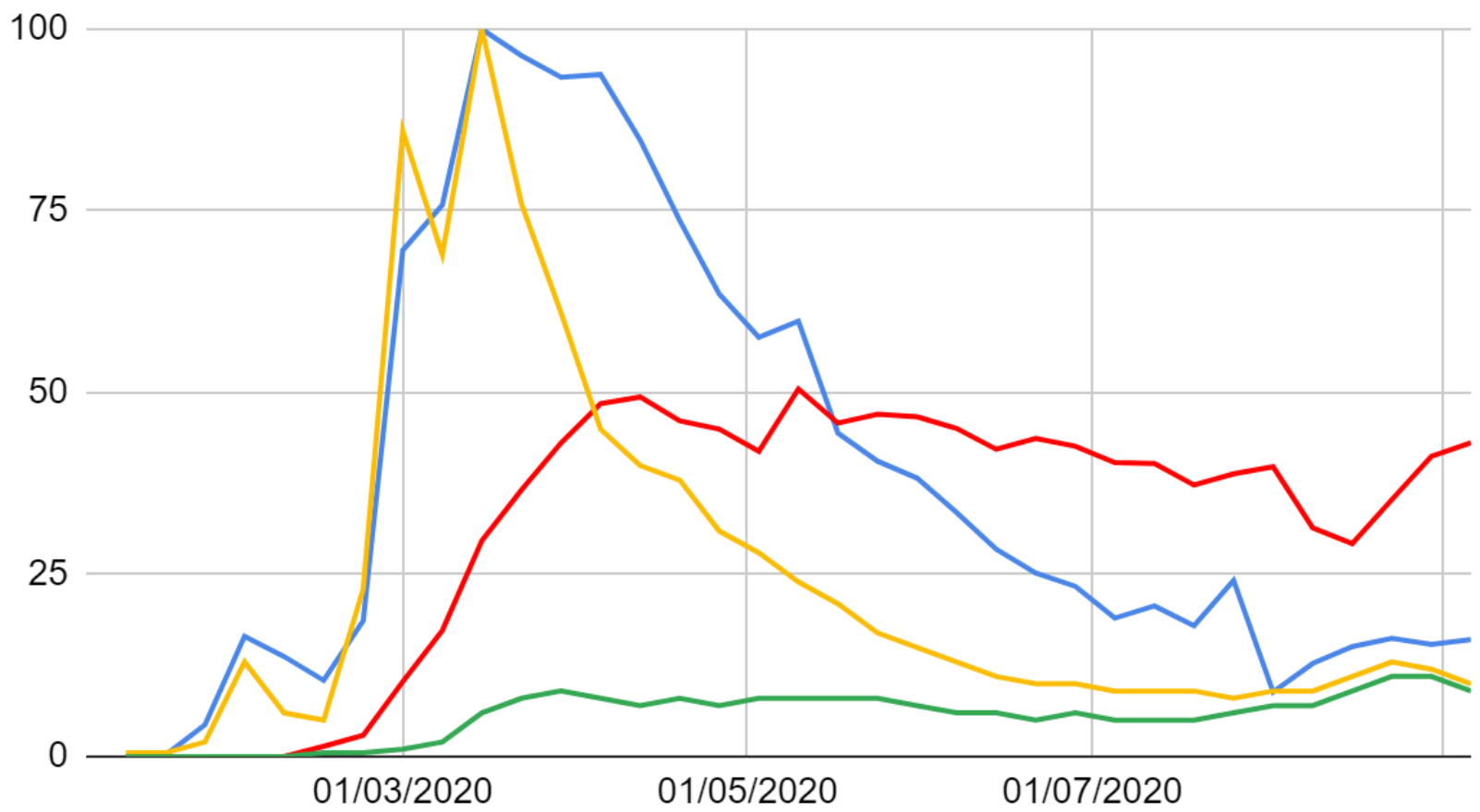

Figure 1. Comparison between weekly RSVs of the keywords "coronavirus" (yellow) and "covid" (green) with the number of times the latter have been adopted by the newspaper "La Repubblica" (blue and red, resp.) from January 1 to September 6, 2020. All values were normalized to 100. RSV = Google Trends relative search volume, t.d.i = titles daily increase.

In the period from the week "January 13-19" to that of the peak "March 9-15", the average percentage discrepancy between all data pairs was significantly smaller than in the following months up to August $\left(\Delta_{1, a v} \%=68 \pm 6, \Delta_{2, a v}=115 \pm 11, t=3.8\right)$. By restricting the analysis to the period 12 January - 12 March 2020 so as to use daily cumulative values, 5 trends with significantly correlates were observed (Figure 2): $r_{1}=.95, P_{1}<.001 ; r_{2}=.78, P_{2}<.001 ; R_{3}=.93, P_{3}=.008 ; R_{4}=$ $.94, P_{4}<.001 ; R_{5}=.94, P_{5}<.001$. From 12 March onwards, a single decreasing trend with a significant correlation was observed until the week of 2-8 August $\left(r_{6}=.97, P_{6}<.001\right)$. 


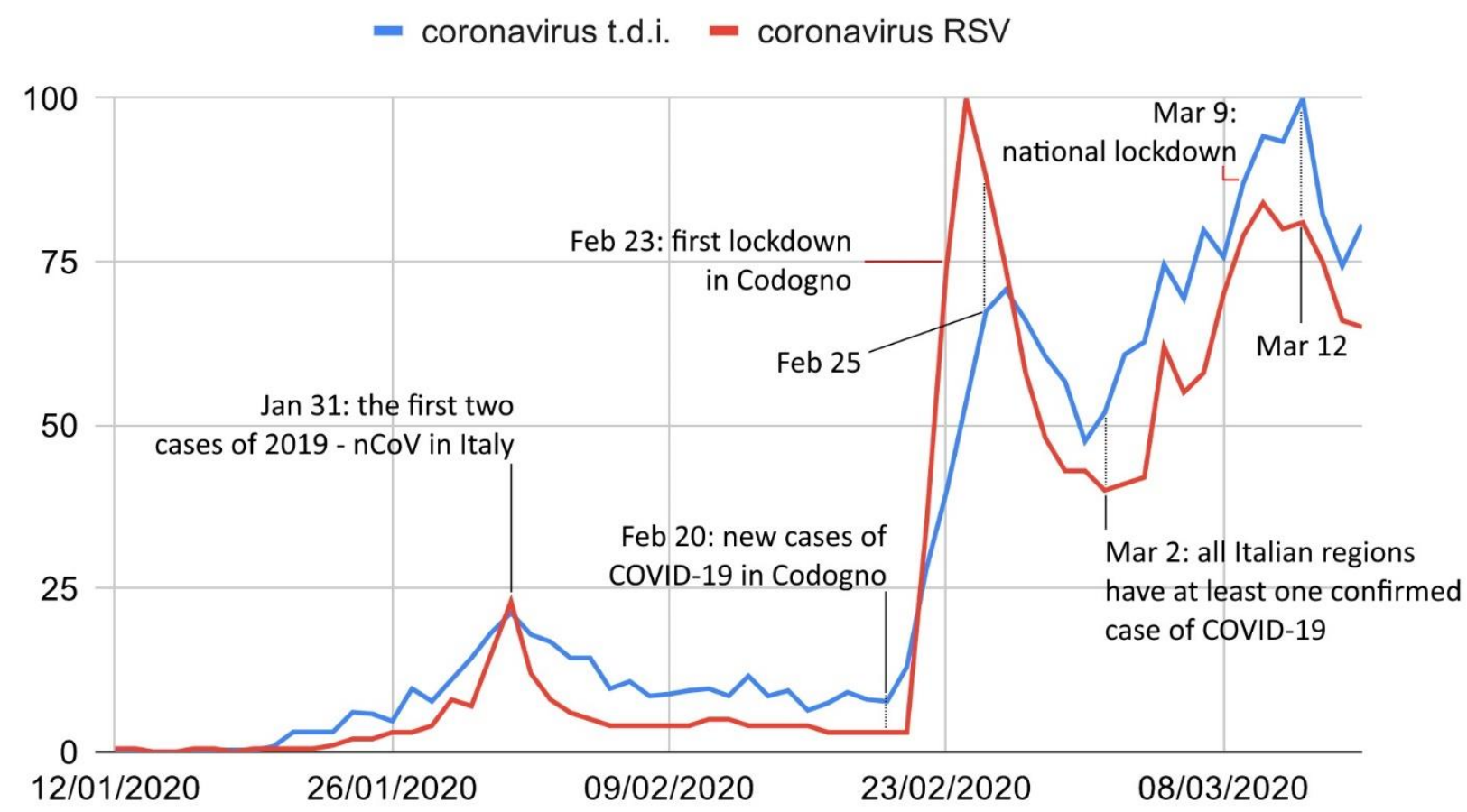

Figure 2. Comparison between daily RSVs (red) of the keywords "coronavirus" and "covid" with the number of times the latter have been adopted by the newspaper "La Repubblica" (blue) from January 12 to March 12, 2020. All values were normalized to 100 . The black lines represent the beginning or the end of a trend. RSV = Google Trends relative search volume, t.d.i = titles daily increase.

As for "Il Corriere della Sera", the results were slightly different: first, the percentage discrepancies between "coronavirus" RSV and t.d.i. were statistically confident $\left(\Delta_{1, a v} \%=99 \pm 13, \Delta_{2, a v}=92 \pm\right.$ $9, t=0.5)$; second, one correlation among the six investigated was weak $\left(r_{1}=.61, P_{1}=.037 ; r_{2}=\right.$ $.56, P_{2}=.013 ; R_{3}=.91, P_{3}=.012 ; R_{4}=.24, P_{4}=.604 ; R_{5}=.57, P_{5}=.067, r_{6}=.96, P_{6}<$ $.001)$.

Since the introduction of the scientific names "SARS-CoV-2" and "COVID-19", the use of the moderately infodemic and non or slightly infodemic names has partially changed compared to the previous period (Table 2). However, even in this case, all the media are characterized by greater use of the generic name "coronavirus".

\begin{tabular}{rrrrr}
\hline & sars-cov-2 & covid (and related) & Novel coronavirus & coronavirus \\
\hline Infodemic Scale & 0 & 1 & 1 & 4 \\
\hline PubMed & $27.5 \%$ & $38.6 \%$ & $28.8 \%$ & $5.1 \%$ \\
\hline Ministry of Health & $1.3 \%$ & $78.2 \%$ & $9.0 \%$ & $11.5 \%$ \\
\hline Google & $24.3 \%$ & $28.4 \%$ & $25.7 \%$ & $21.6 \%$ \\
\hline La Repubblica & $0.6 \%$ & $33.2 \%$ & $1.3 \%$ & $64.8 \%$ \\
\hline Other Newspapers ${ }^{*}$ & $1.2 \%$ & $44.0 \%$ & $1.0 \%$ & $53.8 \%$ \\
\hline Rai (Google News) & $1.6 \%$ & $39.9 \%$ & $1.4 \%$ & $57.1 \%$ \\
\hline Mediaset (Google News) & $0.0 \%$ & $15.4 \%$ & $0.6 \%$ & $84.0 \%$ \\
\hline
\end{tabular}

Table 2. Rate of adoption of COVID-19-related terms during the lockdown (Feb 11 - May 18, 2020). 
In the period 11 February - 18 May 2020, the denominations "SARS-CoV-2" and "COVID-related" were used differently by Italian users $\left(\forall R S V<1\right.$ vs $R S V_{a v}=64$, respectively). "COVID-related" terms were used less frequently than "coronavirus" until the second week of September $\left(\Delta_{R S V, a v} \%=\right.$ $404, t=5.0)$. Until the second week of May, the weekly adoption of "COVID-related" terms by "La Repubblica" was significantly lower than that of the term "coronavirus" $\left(\forall \Delta_{\mathrm{i}} \% \in[642,18]\right)$. Furthermore, the percentage discrepancies between "COVID-related" RSV and t.d.i. were substantial $\left(\Delta_{a v} \%=509 \pm 32\right.$ in the whole investigated period). Nonetheless, correlations were found in the period from the week 10-16 February to 23-29 March $\left(r_{1}=.99, P_{1}<.001\right)$ and in the period from the week 23-29 March to 20-26 July $\left(r_{2}=.64, P_{2}=.007\right)$. Finally, among all the COVID-related terms, "covid" was the one most exploited by users despite the more appropriate and specific "covid19 " $\left(R S V_{a v}=47\right.$ vs $\left.R S V_{a v}=2\right)$. The results for "Il Corriere della Sera" were similar $\left(\Delta_{R S V, a v} \%=\right.$ $\left.773 \pm 69, R_{1}=.97, P_{1}<.001 ; R_{2}=.49, P_{2}=.037\right)$.

Therefore, if a causal correlation of the type "newspaper titles $\rightarrow R S V$ " did exist, three pieces of evidence are highlighted: i) it caused a variation of the RSV highly similar to that of the titles of "La Repubblica" and "Il Corriere della Sera" before the peak, ii) it caused a variation of the RSV more pronounced after the peak (for small variations of the independent variable, large variations of the dependent variable were observed), iii) it substantially influenced the Italian web users in the adoption of the terms used to identify SARS-CoV-2, generating a dangerous disparity between scientific and more infodemic denominations.

\section{Evidence supporting causation}

Non-causal correlations could be due to a) stochastic phenomena, and b) other triggering phenomena (spurious correlations). We found up to 6 significant consecutive trends $\left(M K P_{a v}=.007 \pm .006\right.$, $\forall P<.04)$ and as many strong correlations. Suppose that the correlations found are due to chance (hypothesis "a") and their probability of occurring is $50 \%$ (which is a large overestimate since it would mean that, generating random data, the probability of obtaining a monotonous trend is the same as getting heads by flipping a coin). The probability of obtaining such a scenario from chance is given by $(0.5)^{6}<.016$. A more realistic estimate was obtained by generating random values using the "Random Generator Number" provided by Dr. Haahr [23]: in particular, by generating 50 data series composed of 6 numbers and looking for 1225 Pearson and Spearman correlations between them, significant correlations (i.e. close to the threshold $\alpha=.05$ ) were obtained in about $6.5 \%$ of cases; consequently, the probability of obtaining 6 consecutive significant correlations was $(6.5 / 100)^{6}<$ $.10^{-6}$. Furthermore, the hypothesis investigated was targeted thus avoiding the problem of the "lookelsewhere" effect. Such targeting was based on data of this paper (e.g. the rate of adoption of COVID19-related terms) and previous works $[11,12,10]$. Hence, it is legitimate to consider these correlations not due to chance. Now suppose that both RSVs and newspaper titles were causally influenced by a third quantity $x$. Surely, this quantity must have been linked to the COVID-19 epidemic; ergo, we started searching for correlations between RSV and COVID-19 cases (including deaths, hospitalizations, etc.). Considering the time-lapse 20 February and 12 March 2020, we found three distinct groups of correlations: $r \in[.97, .98], P \in[.004, .005]$ in the period 20-24 February, $R \in$ $[-.83,-.91], P \in[.004, .02]$ in the period 24 February to 1 March, and $R \in[.86, .92], \forall P<.001$ in the period 2-12 March. After that, although COVID-19 cases continued to rise until April, RSV 
started declining as did the "coronavirus" newspaper titles. This allows us to preliminarily conclude that web users have been more influenced by the media rather than by the trend of COVID-19 cases. Nonetheless, linear regression and multiple regression analyzes were performed between the quantities COVID-19 cases, newspaper titles, RSV following the scheme shown in Figure 3 (next page). These results also suggest a greater influence of newspapers towards RSV, although there is a marked dependence between all the variables. Furthermore, by improperly assuming independence between the normalized variables COVID-19 cases $\left(X_{1}\right)$ and Newspapers titles $\left(X_{2}\right)$ and calculating an LSM multiple regression plan for the $R S V(\hat{y})$, we got $\hat{y}=16 X_{1}+1.7 X_{2}-17$ and $\hat{y}=0.05 X_{1}+$ $X_{2}-15$.

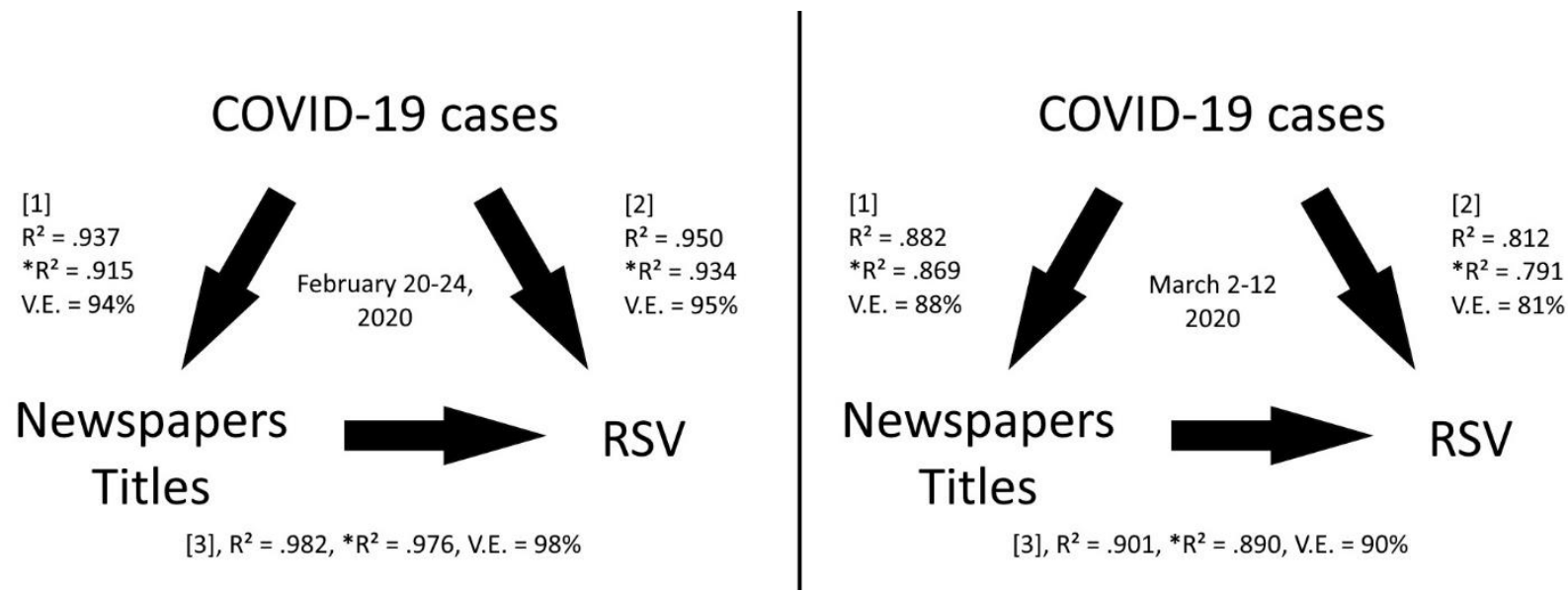

Figure 3. Causal implications scheme and linear regression results. V.E. = variability explained, RSV = Google Trends relative search volume.

Although it is glaring that, in some cases, both COVID-19 cases and newspapers have contributed to conditioning Italian web users, COVID-19 cases had a more short-term and discontinuous effect than newspaper titles. Anyway, it must be considered that even this kind of news is disseminated by disclosure sources which can be I) official government sources (i.e. the Italian Ministry of Health and the Higher Institute of Health), II) private websites, blogs, and social networks, or III) media. Thus, let us start by assuming that all citizens, once they have become aware of the COVID-19 issue, have consulted official sources (hypothesis "I"). Considering that these used, in the vast majority of cases, the less infodemic names (83.5\%), and the news they reported were few and not correlated with RSV (e.g. the Ministry of Health produced 91 articles from 1 January to 12 March against tens of thousands by media), we can exclude this hypothesis. Suppose then that the RSV was influenced by unofficial sources not directly related to press and newscasts (hypothesis "II"). Since press and newscast have their independent sources and we already excluded every random correlation as well a global correlation with COVID-19 cases, we can only suppose a causal global process of the type "media $\rightarrow$ private websites, blogs, social networks $\rightarrow R S V$ " which confirms the fundamental role of media. Therefore, the only hypothesis with empirical evidence is that media, such as press and newscasts, have predominantly determined not only the web searches' trends but also the terms used to carry out such searches (hypothesis "III"). 


\section{COVID-19 symptoms-related web searches}

As happened for the queries previously investigated, the keyword "coronavirus symptoms + covid symptoms" in the timelapse January 20 - May 23 has been influenced by media $\left(\rho_{a v}=.92 \pm\right.$ $.03, \forall P<.007)$. Its RSV was highly sensitive to news, reaching peaks of $74 \pm 0.7,86 \pm 0.8$, and $100 \pm 0.1$ on January 31, February 23, and March 9, respectively. Among the COVID-19 symptoms reported by the Italian Ministry of Health, the ones that got the most web searches during the whole pandemic were fever $(R S V=49 \pm 4.6)$, cough $(23.0 \pm 3.5)$, cold $(15.2 \pm 2.2)$, sore throat $(12.5 \pm$ $1.3)$, and pneumonia (12.3 \pm 3.2$)$. During the previous 3 years (2017-2019), these showed winter seasonalities but not global nor local trends or unit-roots $(\forall A D F P<.004, \forall m<0.05$, supplementary figure 1). This allowed us to easily identify two anomalies: February-May 2020, with $\Delta_{\text {peak }} \% \in[50,835], t \in[14,21]$, and August-November 2020, with $\Delta_{\text {peak }} \% \in[46,264], t \in$ $[2.8,6.2]$ (except for "cough"). Therefore, the impact of the pandemic on this type of search has been substantial. Nonetheless, there is evidence that counteracts the potential use of such web searches to predict COVID-19 cases: in fact, the general RSV trend in the period February - May 2020 presents more similarities and correlations with newspaper titles than with COVID-19 cases (Figure 4).

- fever - cough - sore throat - cold - pnemuonia $=$ "coronavirus" t.d.i.

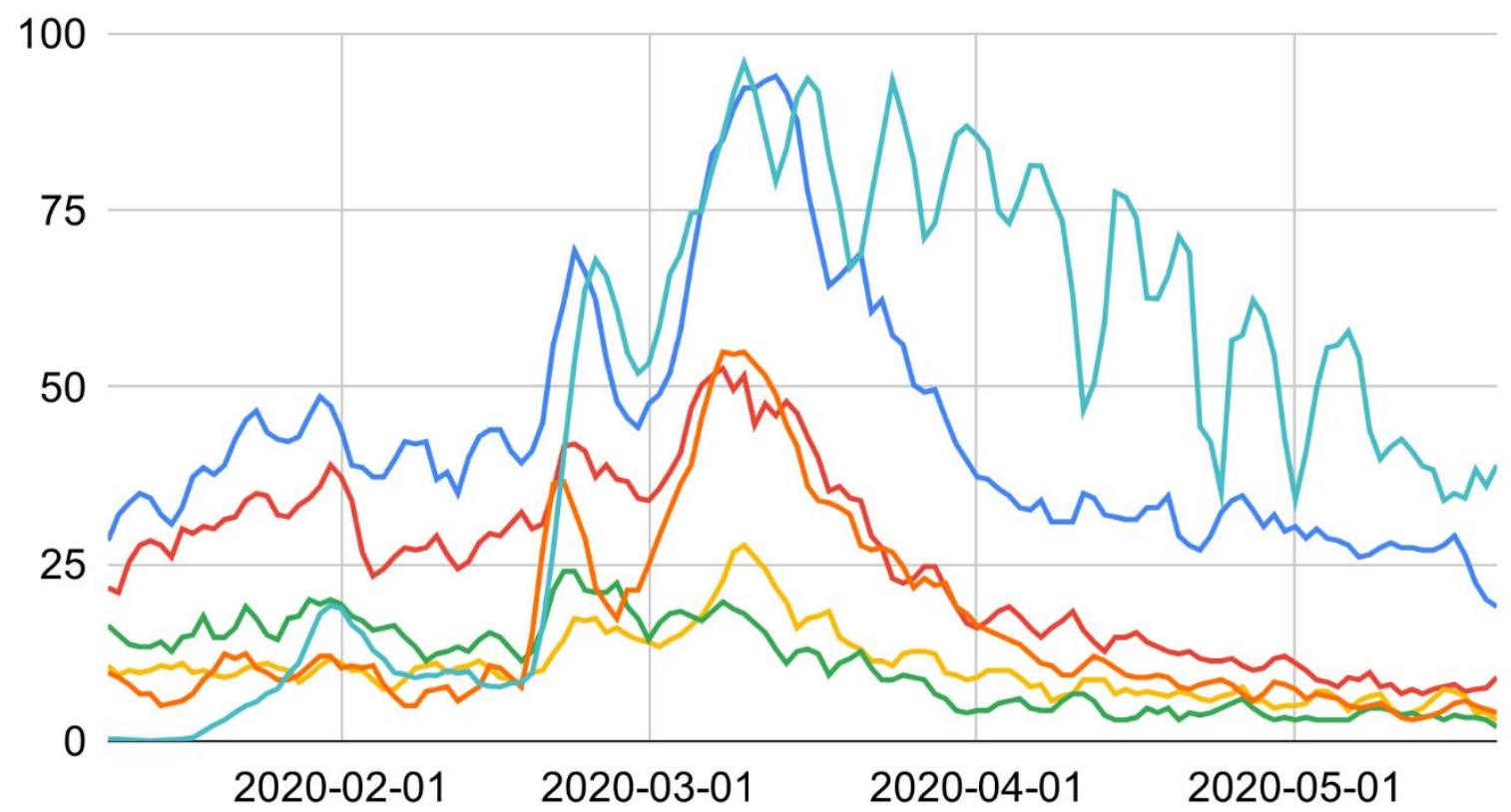

Figure 4. Comparison between COVID-19 symptoms-related web queries (Google Trends relative search volumes) and "La Repubblica" newspaper titles containing the word "coronavirus" from January to May 2020.

In particular, although the analysis of cross-correlations has identified some optimum values with a lag of 2 days, the onset of local RSV trends never preceded that of newspaper headlines. Additionally, we once again observed two clear decreasing trends in RSV between late February and early March and after 15 March, despite the continued increase in COVID-19 cases. 


\section{COVID-19 web interest during the second wave}

The "coronavirus + covid" query had a near-stationary RSV between early June and early August $(M K P=.135, S=-0.09, S D \%=8.3 \%)$. Understandably, because of the sharp decrease in COVID-19 cases and the easing of containment measures, this was much less pronounced than during the first wave $(\Delta \%=-71.4, t=-14.2)$. From August 2020 to March 17, 2021, web interest in COVID-19 remained significantly lower than that of the first lockdown $(\Delta \%=-57.8, t=-4.2)$, showing spikes of RSV at the start of the second wave ( $R S V_{\max } \pm 0.4$, August 2020), a steep rise of cases from 1 October 2020 until about mid-November $\left(R S V_{\max }=35 \pm 0.7\right)$, and mid-February, anticipating the increase in cases at the end of February by about 10 days $\left(R S V_{\max }=23 \pm 0.5\right)$. The use of the term "covid" has surpassed that of the more generic and infodemic "coronavirus" (59\% vs 41\% from September 2020 to March 2021). On the contrary, the more technical "SARSCoV-2" has not been adopted by web users $(\forall R S V<1)$. This time, we identified strong differences in the adoption of COVID-19-related terms between newspapers and other sources such as "Rai" and "Mediaset" (Table 3).

\begin{tabular}{rrrrr}
\hline & sars-cov-2 & covid (and related) & Novel coronavirus & coronavirus \\
\hline Infodemic Scale & 0 & 1 & 1 & 4 \\
\hline PubMed & $26.6 \%$ & $42.9 \%$ & $27.6 \%$ & $2.8 \%$ \\
\hline Ministry of Health & $0.9 \%$ & $90.9 \%$ & $1.8 \%$ & $6.4 \%$ \\
\hline Google & $25.7 \%$ & $28.6 \%$ & $22.9 \%$ & $22.9 \%$ \\
\hline La Repubblica & $0.9 \%$ & $76.5 \%$ & $0.3 \%$ & $22.3 \%$ \\
\hline II Corriere d. Sera & $1.5 \%$ & $75.1 \%$ & $0.6 \%$ & $22.7 \%$ \\
\hline Other Newspapers* & $1.1 \%$ & $74.0 \%$ & $0.1 \%$ & $24.8 \%$ \\
\hline Rai (Google News) & $0.5 \%$ & $49.3 \%$ & $0.0 \%$ & $50.2 \%$ \\
\hline Mediaset (Google News) & $0.0 \%$ & $38.6 \%$ & $1.3 \%$ & $60.1 \%$
\end{tabular}

Table 3. Rate of adoption of COVID-19-related terms during the lockdown (May 19, 2020 - March 17, 2021).

Despite the use of COVID-related terms by newspapers exceeded that of the generic name "coronavirus" in May 2020, the web users showed a delay of 4 months to do the same. Finally, web users' interest in COVID-19 decreased by about $20 \%$ compared to the first wave.

\subsection{Media influence on COVID-19 Infodemic}

\section{YouTube videos}

The YouTube channels of the following news broadcasts were investigated (the number of subscribers is shown in brackets): Rai (4.08 million), LA7 Attualità (730,000), MediasetPlay (605,000), La Repubblica (576,000), Corriere della Sera (145,000), and Tgcom24 (52,700). Only videos with over than 100,000 views were considered for analysis (the number of views is shown in brackets). The views of videos containing the keyword "coronavirus" overwhelmed those containing the keywords "COVID-related" and "sars-cov-2" (50.07 million vs 8.51 million, $\delta \%=141.9$ ). Moreover, some videos with the most views had extremely infodemic titles, including: "Covid does 
not exist" $\left(621,945,1^{\text {st }}\right.$ on La7 Attualità), "The 2015 Rai-Leonardo video on the virus created in China in the laboratory. The scientific community..." $\left(401,648,4^{\text {th }}\right.$ on Il Corriere della Sera, "Coronavirus, Vittorio Sgarbi: "It is not an epidemic because there is no risk of death" $\left(440,296,2^{\text {nd }}\right.$ on La7 Attualità), "Coronavirus, Sgarbi: The confinement of asymptomatic people is fascism" (396,144, ), "Coronavirus, the conference of "deniers" in the Senate: The virus in Italy is no more"(189,655, 47 $7^{\text {th }}$ on La Repubblica), "Covid, Prof. Roberto Bernabei: It's a normal disease..." $\left(141,233,21^{\text {th }}\right.$ on La7 Attualità), "Coronavirus, the reassurance of the infectious disease specialist Matteo Bassetti: It's not an infection..." (the video talks about similarities with the flu, 203,347, 14 ${ }^{\text {th }}$ on La7 Attualità). Besides, we report the presence of growing interest in information channels that discloses serious fake news, such as ByoBlu (524,000 subscribers). In particular, this channel often shares scientifically unjustified opinions of people famous for their conspiratorial positions on COVID-19. However, an exact estimate is difficult to make as many videos have been blacked out by YouTube for disinformation [24].

\section{Web searches}

Some statements of prominent personalities, including scientists, have directed the web interest of users towards disinformation and misinformation (Figure 5).

- Sgarbi - Zangrillo - Montagnier - Laboratory - Vaccines Side Effects

- Montanari

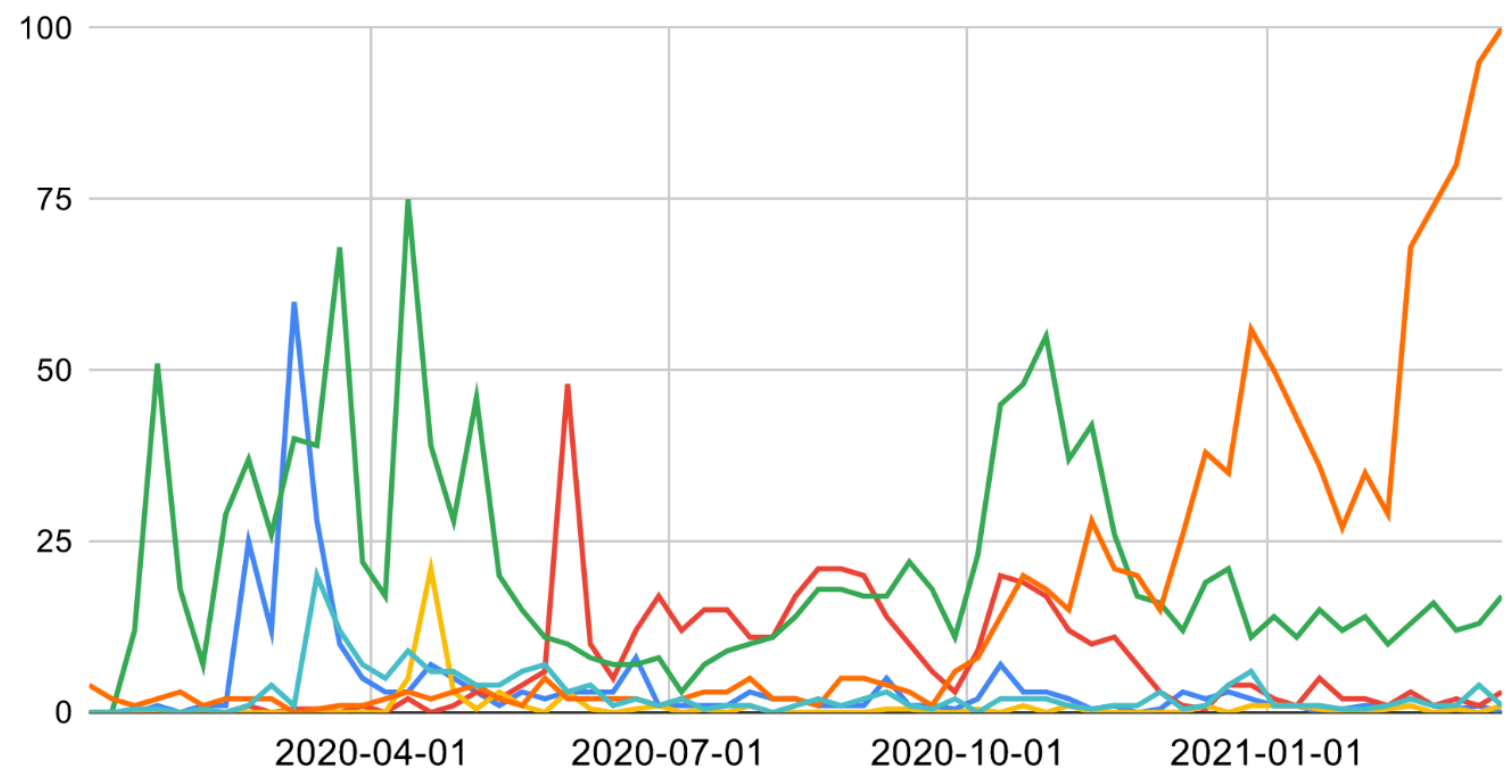

Figure 5. Infodemic query RSVs since the start of the pandemic. The "astrazeneca" query is not shown in order to allow you to view the other RSV trends.

Among these, on February 23, 2020, Dr. Maria Rita Gismondo compared COVID-19 to a seasonal flu [25]. On the same day, the RSV of the "coronavirus flu + covid flu" query went from 0 to 100 , maintaining high values until 22 March $\left(R S V_{a v}=40.2 \pm 3.2\right)$. On February 24, 2020, politician 
Vittorio Sgarbi minimizes the risk of death from COVID-19 without any supporting scientific evidence [26]. On the same day, the proper RSV of the "coronavirus sgarbi + covid sgarbi" query went from 2 to 75 . Moreover, Sgarbi became the protagonist of a long series of infodemic statements, including the incitement to violate the anti-COVID-19 regulations [27]. Over the same period, the RSV from the previous query remained high, hitting two new maxima on 10 and 14 March (PSVs = $100, P S V=93$, respectively). Other major fake news circulated through the media involved the creation of COVID-19 in a Chinese laboratory. The following contributed to this phenomenon: i) a 2015 report by a local news program, reproposed by the media, ii) the words of the politician Matteo Salvini, and iii) the statements of the Nobel Prize winner Luc Montagnier [10, 28]. Another promoter of conspiracy theories was Dr. Stefano Montanari [29]. Corresponding to his statements, there was a spike in the RSV of the query "montanari coronavirus + montanari covid". On May 31, 2020, Dr. Zangrillo declared the disappearance of COVID-19 [30]. The same day, there was a heavy rise in the query "coronavirus zangrillo + covid zangrillo". However, the major infodemic impact by the media has been towards vaccines, especially for the "AstraZeneca" vaccine. Figure 5 shows a clear trend in the weekly RSV of the query "vaccines side effects" from the second week of February 2021 onwards ( $S S=10, M K P=.009$ ). By narrowing the range to get daily RSVs, a clear level shift can be observed from 12 to 14 February 2021. In particular, comparing the periods January 20 - February 13 and February 17 - March 9, we observed a net percentage increase in web interest $(\Delta \%=205.7 \%$, $t=17.4)$. However, the peak is reached on March $11(R S V=100)$, with a sharp rise starting on March 9. In the same period, a long series of totally misleading headlines were published by the main Italian newspapers, fomenting the distrust of vaccines [31,32]. Finally, comparing the same periods, the RSV of the query "astrazeneca side effects" has had an extreme increase $\Delta \%=2991.4 \%, t=$ 16.9 . 


\section{Discussion}

The results of this paper suggest that the main Italian mass media have heavily influenced not only the trend of web interest in COVID-19, but also the terms adopted by users to identify the virus. In particular, there was a pronounced disparity between the use of scientific names and the more infodemic ones: official sources, such as the Ministry of Health or international medical databases such as PubMed, used non or little infodemic terms such as "2019 n-cov" (I-scale = 0), "COVID-19" (I-scale = 0), "COVID" (I-scale = 1), "novel coronavirus" (I-scale = 1); on the contrary, in the early stages of the pandemic the mass media used the term "coronavirus" (I-scale $=4$ ) in about $90 \%$ of cases, while from the second half of May 2020 onwards the "COVID-related" names became the most adopted in most cases. Exceptional cases are those of RAI and Mediaset, in which the term "coronavirus" remained the most used. Alongside this, when SARS-CoV-2 was raging in China and the first Italian cases arose, the Italian mass media often referred to the virus with extremely infodemic terms such as "Chinese coronavirus" (I-scale $=8$ ) or "Chinese virus" (I-scale $=9$ ), while the query "2019-ncov" did not produce any results in the RaiPlay and MediasetPlay search engines. In the same period, strong phenomena of racism towards Chinese people in Italy were born [9]. The correlation analysis showed that, during the early stages of the pandemic, the relative search volume trends of the same keywords were essentially determined by that of the newspaper headlines on the same topics. Furthermore, the web user responded with a delay of about 4 months to equalize the use of the terms "COVID-related" with that of the generic name "coronavirus". Queries related to the symptoms of the disease also had a trend comparable to that of the newspaper headlines on COVID-19. Even concerning to mere infodemic, the role of the media was impacting: indeed, often giving a voice to characters with no medical or epidemiological skills and doctors with conspiratorial positions unjustified by scientific literature, they fed a climate of mistrust towards health authorities and antiCOVID-19 measures, both pharmacological and non-pharmacological. As proof of this, peaks in the relative search volume of queries on the same topic and/or containing the name of the person concerned were detected in conjunction with the aforementioned statements, interviews, and article titles. Moreover, some among the most viewed videos on YouTube channels of the news broadcaster had highly infodemic headlines. Specifically, the topics of greatest interest to the media and the public were: i) creation of the virus in a Chinese laboratory, a thesis supported by prominent politicians such as Matteo Salvini and even by the Nobel Prize winner Luc Montagnier, but with no scientific evidence, ii) the minimization of the COVID-19 crisis, a hypothesis supported by the politician Vittorio Sgarbi but also by Dr. Maria Rita Gismondo who compared the disease to seasonal flu without any scientific evidence, and iii) the disappearance of COVID-19, a hypothesis supported by Dr. Alberto Zangrillo without any scientific evidence. Finally, the "AstraZeneca" vaccine question deserves a separate chapter. The European Medicines Agency authorized Astra Zeneca's SARS-CoV2 vaccine for use in all adults aged over 18 on 29 January 2021 [36]. The first concerns about its inoculation arose with the suspension in several European countries, including Italy, in early March 2021 [37]. Although it was specified that these suspensions were merely precautionary since due to some reports of possible thromboembolic events, many Italian newspapers used unnecessarily alarmist and terrifying headlines as reported by the website "Bufale.net" (a private Italian factchecking portal against disinformation, hoaxes and alarmism that are rampant on the Internet) [31]. Thus, although the vaccine has now been re-approved by the European Medicine Agency [38], queries about its possible side effects have an increasing trend as shown in Figure 5. Therefore, the answers to questions R1, R2, R3 and R4 are respectively: R1) the Italian media have substantially 
influenced the Italian web user in the adoption of terms used to identify the novel coronavirus (COVID-19 disease), R2) the Italian media have substantially influenced the web interest of Italian users in the novel coronavirus (COVID-19 disease), R3) the Italian media have contributed in a not inconsiderable way to the spread of fake news and unjustified alarmism related to the novel coronavirus (COVID-19 disease), and R4) Google Trends has shown great limitations as a predictive tool of possible epidemiological situations as the web user has been strongly addressed by the mass media also and even in the search for COVID-19 symptoms.

\section{Limitations}

This analysis was subject to some limitations. First, there are no guarantees that the interest of Italian web users can well represent the interest of the entire Italian population, limiting the conclusions of this paper to Italian Internet users. Furthermore, causal correlations were only searched between Google Trends relative search volume and the titles used by the two main Italian newspapers. Future research could investigate the correlations between the Italian population and the mass media, also involving non-netizens and all Italian media. Finally, the paper cannot represent the entire totality of the interests of web users that arose during the COVID-19 pandemic.

\section{Conclusions}

Since the main Italian mass media have strongly influenced the perception of risk and interest of Italian web users towards the novel coronavirus (COVID-19 disease), we suggest that the Italian authorities put strict and effective controls on the information circulating in Italy. Furthermore, the authors of this paper invite the directors of the main Italian newspapers and newscasts to stick to the scientific denominations of SARS-CoV-2 or any other future virus or disease, better pondering the choice of article titles so as not to foment the infodemic. Finally, the authors of this paper recommend carefully weighing the influence of mass media on users' web searches before adopting any epidemiological predictive models based on Google Trends or similar infoveillance tools. 


\section{Methods}

\subsection{Design}

To evaluate the impact of the media on Italian users' web interest, we compared the use of specific keywords by the main Italian media with the relative search volume (RSV) of the same keywords on Google Trends, looking for: i) significant and substantial correlations and cross-correlations between RSVs and the article titles of the two most read newspapers, ii) triggering events, i.e. events that triggered a trend (e.g. the beginning of a trend of the query "the covid does not exist" in conjunction with related public statements reported by newspapers), and iii) similarities and differences in keyword adoption rates.

\subsection{Data collection}

Given the heterogeneity of the data collected, different methods were used for each type of source investigated. Specific keywords were searched in specific periods according to the following scheme:

- January 1 - February 13, 2020 (period 1): 2019-ncov, novel coronavirus, coronavirus, chinese virus, chinese coronavirus.

- February 11 - May 18, 2020 (period 2): sars-cov-2, covid-related (i.e. covid, covid 19, covid19, covid-19), novel coronavirus, coronavirus

- May 19, 2020 - March 17, 2021 (period 3): sars-cov-2, covid-related (i.e. covid, covid 19, covid19, covid-19), novel coronavirus, coronavirus

Given the diversity of the search algorithms of the investigated platforms, different selection criteria were adopted in order to make the results comparable.

- PubMed: the results of each query were counted. Since the search engine provided intersections in the results for the "coronavirus" $(N)$ and "novel coronavirus" $(n)$ queries, the $N-n$ calculation was performed to estimate the exact number of the former.

URL: https://pubmed.ncbi.nlm.nih.gov/

- Ministry of Health: the number of official press releases containing each specific query was counted.

URL:http://www.salute.gov.it/portale/nuovocoronavirus/archivioComunicatiNuovoCoronav irus.jsp?lingua $=$ italiano $\& a n n o=2020 \& a r e a=$ nuovocoronavirus $\&$ comunicati.page $=4$

- Google: the number of results containing each specific query was counted.

URL: https://www.google.it/

- La Repubblica: data was collected from the official archive. The titles containing each of the specific keywords have been counted. The "advanced search" and "exact search" filters were used.

URL: https://ricerca.repubblica.it/repubblica/archivio/repubblica/

- Il Corriere della Sera: data was collected from the official archive. The titles containing each of the specific keywords have been counted. Since the search engine provided intersections in the results for the "coronavirus" $(N)$ and "novel coronavirus" $(n)$ queries, the $N-n$ calculation was performed to estimate the exact number of the latter. The "free search" filter was used. 


\section{URL: http://archivio.corriere.it/Archivio/interface/landing.html}

- Titoli Giornali (Other Newspapers): data was collected from the official archive. The titles containing each of the specific keywords have been counted.

URL: https://www.titoligiornali.it/

- Rai (Google News): the number of results containing each specific query was counted. The term "rai" has been added to each query. The selected item was "Google News".

URL: https://www.google.it/

- Mediaset (Google News): the number of results containing each specific query was counted. The term "rai" has been added to each query. The selected item was "Google News".

URL: https://www.google.it/

- YouTube: the number of results containing each specific query was counted. Each query was accompanied by the name of the YouTube channel investigated. The filter "sort by number of views" was used.

URL: https://www.youtube.com/

- Google Trends. All data on the searched queries was downloaded in the ".csv" file format. The exact list of keywords is shown in section 2 (Results).

URL: https://trends.google.it/trends/

\subsection{Statistical analysis}

\section{Linear regression}

When data were normally distributed, the angular coefficient $(m)$ of the interpolating line was calculated to evaluate the importance or absence of a trend. Moreover, Pearson $(R)$ and adjusted Pearson $(* R)$ coefficients were also calculated. Finally, the percentage of variability of the dependent variable explained by the independent variable $(V . E$.) was calculated.

\section{Mann-Kendall test}

To highlight the presence of trends within a dataset, after an initial graphical analysis, the MannKendall test $(M K)$ was used. Furthermore, the importance of the trend was evaluated with the Sen's slope $(S S)$.

\section{Mean values}

The mean value of a data series " $X$ " was indicated with " $x_{a v}$ ". All average values were presented in the form " $x_{a v} \pm S E M$ ", where "SEM" indicates the standard error of the mean. These measurements were used when the datasets were normally distributed or containing at least 30 elements [33]. The variability of a dataset was evaluated through the percentage standard deviation $(S D \%)$, calculated as the ratio between the standard deviation and the mean value multiplied by 100 .

\section{Percentage increases}

The percentage increases between two time-dependent values were calculated with the following formula: 


$$
\Delta \%=[x(t+|\Delta t|)-x(t)] / x(t) \cdot 100 .
$$

When the values did not depend on time, the following formula was used:

$$
\Delta \%=\left(x_{2}-x_{1}\right) / \min \left\{x_{1}, x_{2}\right\}
$$

\section{Percentage differences}

The percentage difference between two values was calculated with the formula:

$$
\delta \%=2\left|x_{2}-x_{1}\right| /\left(x_{1}+x_{2}\right) .
$$

\section{Pearson and Spearman correlations}

When the datasets were normally distributed, Pearson's correlation $(R)$ was used; otherwise, Spearman's $(r)$ was used. The correlation strength was assessed independently of the P-values. When arithmetic averages containing both correlations were calculated, the notation " $\rho_{a v}$ " was used.

\section{P-values and significance threshold}

P-values were used as a continuous measure of evidence against the null hypothesis [34]. However, an indicative significance threshold of $5 \%(\alpha=.05)$ was set in all tests.

\section{Shapiro-Wilk test}

To evaluate the distributive normality of a dataset, the Shapiro-Wilk test combined with a graphical analysis was used.

\section{Welch's t-test}

When the datasets were found to be normally distributed, Welch's t-test $(t)$ was used. Furthermore, when the size of the analyzed sets exceeded 30 elements, the central limit theorem was exploited to use Welch's test even with data not normally distributed [35]. Two values were judged to belong to different distributions approximately when $t>1.9$.

\subsection{Software}

Microsoft Excel 2020 software was used for data analysis. In addition, RealStatistics (https://www.real-statistics.com/) and XLSTAT (https://www.xlstat.com/en/) packages were used. 


\section{References}

[1] Verma, P., Dumka, A., Bhardwaj, A., Ashok, A., Kestwal, M.C., Kumar, P. A Statistical Analysis of Impact of COVID19 on the Global Economy and Stock Index Returns. SN Comput Sci. 2(1):27; 10.1007/s42979-020-00410-w (2021).

[2] Akande, O.W., Akande, T.M. COVID-19 pandemic: A global health burden. Niger Postgrad Med J. 27(3):147-155; 10.4103/npmj.npmj_157_20 (2020).

[3] COVID-19 Dashboard by the Center for Systems Science and Engineering (CSSE) at Johns Hopkins University (JHU). Johns Hopkins University \& Medicine. Accessed: March 21, 2021.

https://coronavirus.jhu.edu/MAP.HTML

[4] Managing the COVID-19 infodemic: Promoting healthy behaviours and mitigating the harm from misinformation and disinformation. World Health Organization (WHO). Accessed: March 21, 2021.

https://www.who.int/news/item/23-09-2020-managing-the-covid-19-infodemic-promoting-healthybehaviours-and-mitigating-the-harm-from-misinformation-and-disinformation

[5] Cinelli, M., et al. The COVID-19 social media infodemic. Sci Rep. 10, 16598; 10.1038/s41598020-73510-5 (2020).

[6] Ferrara, E., Cresci, S., Luceri, L. Misinformation, manipulation, and abuse on social media in the era of COVID-19. J Comput Soc Sc. 3, 271-277; 10.1007/s42001-020-00094-5 (2020).

[7] Gottlieb, M., Dyer, S. Information and Disinformation: Social Media in the COVID-19 Crisis. Acad Emerg Med. 27(7):640-641; 10.1111/acem.14036 (2020).

[8] Islam, M.S. et al. COVID-19-Related Infodemic and Its Impact on Public Health: A Global Social Media Analysis. Am J Trop Med Hyg. 103(4):1621-1629; 10.4269/ajtmh.20-0812 (2020).

[9] Rovetta, A., Bhagavathula, A.S. COVID-19-Related Web Search Behaviors and Infodemic Attitudes in Italy: Infodemiological Study. JMIR Public Health Surveill. 6(2): e19374; 10.2196/19374 (2020).

[10] Rovetta, A., Bhagavathula, A.S. Global Infodemiology of COVID-19: Analysis of Google Web Searches and Instagram Hashtags. J Med Internet Res. Aug; 22(8): e20673; 10.2196/20673 (2020). 
[11] Dagher, S.H., Lamé, G., Hubiche, T., Ezzedine, K., Duong, T.A. The Influence of Media Coverage and Governmental Policies on Google Queries Related to COVID-19 Cutaneous Symptoms: Infodemiology Study. JMIR Public Health Surveill. 7(2): e25651; 10.2196/25651 (2021).

[12] González-Padilla, D.A., Tortolero-Blanco, L. Social media influence in the COVID-19 Pandemic. Int Braz J Urol. 46(Suppl 1): 120-124; 10.1590/S1677-5538.IBJU.2020.S121 (2020).

[13] Milinovich, G. J., Williams, G. M., Clements, A. C. A. \& Hu, W. Internet-based surveillance systems for monitoring emerging infectious diseases. Lancet Infect. Dis. 14, 160-168; 10.1016/S1473-3099(13)70244-5 (2014).

[14] Brodeur, A., Clark, A.E., Fleche, S., Powdthavee, N. COVID-19, lockdowns and well-being: Evidence from Google Trends. J Public Econ. 193: 104346; 10.1016/j.jpubeco.2020.104346 (2020).

[15] Garaus, M., Garaus, C. The Impact of the Covid-19 Pandemic on Consumers' Intention to Use Shared-Mobility Services in German Cities. Front. Psychol. 12:646593; 10.3389/fpsyg.2021.646593 (2021).

[16] Greene, S.K. et al. Nowcasting for Real-Time COVID-19 Tracking in New York City: An Evaluation Using Reportable Disease Data From Early in the Pandemic. JMIR Public Health Surveill. 7(1):e25538; 10.2196/25538 (2020).

[17] Kristensen, K., et al. Exploring the use of web searches for risk communication during COVID19 in Germany. Sci Rep. 11, 6419; 10.1038/s41598-021-85873-4 (2021).

[18] Wong, M.Y.Z., Gunasekaran, D.V., Nusinovici, S., Sabanayagam, C., Yeo, K.K., Cheng, C., Tham, Y. Telehealth Demand Trends During the COVID-19 Pandemic in the Top 50 Most Affected Countries: Infodemiological Evaluation. JMIR Public Health Surveill. 7(2):e24445; 10.2196/24445 (2020).

[19] Google Trends. Google $e^{T M}$. https://support.google.com/trends/

[20] Cervellin, G., Comelli, I., Lippi, G. Is Google Trends a reliable tool for digital epidemiology? Insights from different clinical settings. $J$ Epidemiol Glob Health. 7(3):185-189; 10.1016/j.jegh.2017.06.001 (2017).

[21] Rainews official website. Emergenza coronavirus. Accessed: March 17, 2021. 
https://www.rainews.it/ran24/speciali/2020/covid19/world.php

[22] SkyTg24 official website. Coronavirus. Accessed: March 17, 2021.

https://tg24.sky.it/mondo/coronavirus

[23] Haahr, M. Random.org. School of Computer Science and Statistics. Accessed: March 10, 2021. https://www.random.org/integers/?num=6\&min=1\&max=100\&col=1\&base=10\&format $=$ html

[24] Menichini, R. No vax e complottisti: i social corrono ai ripari contro la disinformazione. La Repubblica. March 13, 2021. Accessed: March 19, 2021.

https://www.repubblica.it/esteri/2021/03/13/news/no_vax_e_complottisti_i_social_corrono_ai_ripar i_contro_la_disinformazione-292022010/

[25] Za, V., Landini, F. Coronavirus clampdown spreads fear and doubt in northern Italy. Reuters. February 23, 2020. Accessed: March 20, 2021.

https://www.reuters.com/article/us-china-health-italy-anxiety/coronavirus-clampdown-spreads-fearand-doubt-in-northern-italy-idUKKCN20H0OS

[26] La7 official website. Coronavirus, Vittorio Sgarbi: "Non è un'epidemia perché non c'è rischio di morte". La7.it. February 24, 2020. Accessed: March 20, 2021.

https://www.la7.it/otto-e-mezzo/video/coronavirus-vittorio-sgarbi-non-e-unepidemia-perche-nonce-rischio-di-morte-24-02-2020-309263

[27] Benedictus, L. No, Italy hasn't recounted its coronavirus data and found that most died from other diseases. Full Fact. May 22, 2020. Accessed: March 20, 2021.

https://fullfact.org/health/sgarbi-coronavirus/

[28] Nardelli, A., D’Urso, J. Italy's Far-Right And Nationalist Leaders Are Pushing Debunked Conspiracy Theories About The Coronavirus To Millions Of Followers. BuzzFeed.News. March 26, 2020. Accessed: March 20, 2021.

https://www.buzzfeed.com/albertonardelli/coronavirus-matteo-salvini-giorgia-meloni-conspiracy

[29] Luna, R. Coronavirus, scienziati italiani denunciano guru online complottista. La Repubblica. March 25, 2020. Accessed: March 20, 2021.

https://www.repubblica.it/scienze/2020/03/25/news/coronavirus_gl_scienziati_italiani_denunciano_ i_guru_online_che gridano_al_complotto-252285764/ 
[30] La Repubblica editorial staff. Coronavirus, Zangrillo: "Il Covid non esiste più, qualcuno terrorizza il paese". Richeldi: "No, il virus circola”. May 31, 2020. Accessed: March 20, 2021.

https://www.repubblica.it/cronaca/2020/05/31/news/coronavirus_zangrillo_il_covid_clinicamente non_esiste_piu_qualcuno_terrorizza_paese-258111004/

[31] "Bufale" editorial staff. "I giornali stupiti per i rifiuti al vaccino AstraZeneca dopo giorni di titoloni su reazioni avverse". Bufale.net. March 14, 2021. Accessed: March 20, 2021.

https://www.bufale.net/i-giornali-stupiti-per-i-rifiuti-al-vaccino-astrazeneca-dopo-giorni-di-titolonisu-reazioni-avverse/

[32] Today.it editorial staff. "AstraZeneca, paura in Europa": polemiche per il titolo di Repubblica. Today.it. March 12, 2021. Accessed: March 20, 2021.

https://www.today.it/rassegna/vaccino-astrazeneca-oggi-repubblica.html

[33] Mascha, E.J., Vetter, T.R. Significance, Errors, Power, and Sample Size: The Blocking and Tackling of Statistics. Anesth Analg. 126(2):691-698; 10.1213/ANE.0000000000002741 (2018).

[34] Amrhein, V., Korner-Nievergelt, F., Roth, T. The earth is flat ( $\mathrm{p}>0.05)$ : significance thresholds and the crisis of unreplicable research. PeerJ. 5: e3544; 10.7717/peerj.3544 (2017).

[35] Kwak, G., Kim, J.H. Central limit theorem: the cornerstone of modern statistics. Korean J Anesthesiol. 70(2): 144-156; 10.4097/kjae.2017.70.2.144 (2017).

[36] Torjesen, I. Covid-19: AstraZeneca vaccine is approved in EU with no upper age limit. BMJ; 372:n295; 10.1136/bmj.n295 (2021).

[37] Wise, J. Covid-19: European countries suspend use of Oxford-AstraZeneca vaccine after reports of blood clots. BMJ. 372:n699 doi:10.1136/bmj.n699 (2021).

[38] Mahase, E. Covid-19: AstraZeneca vaccine is not linked to increased risk of blood clots, finds European Medicine Agency. BMJ. 372:n774; 10.1136/bmj.n774 (2021). 


\section{Supplementary files}

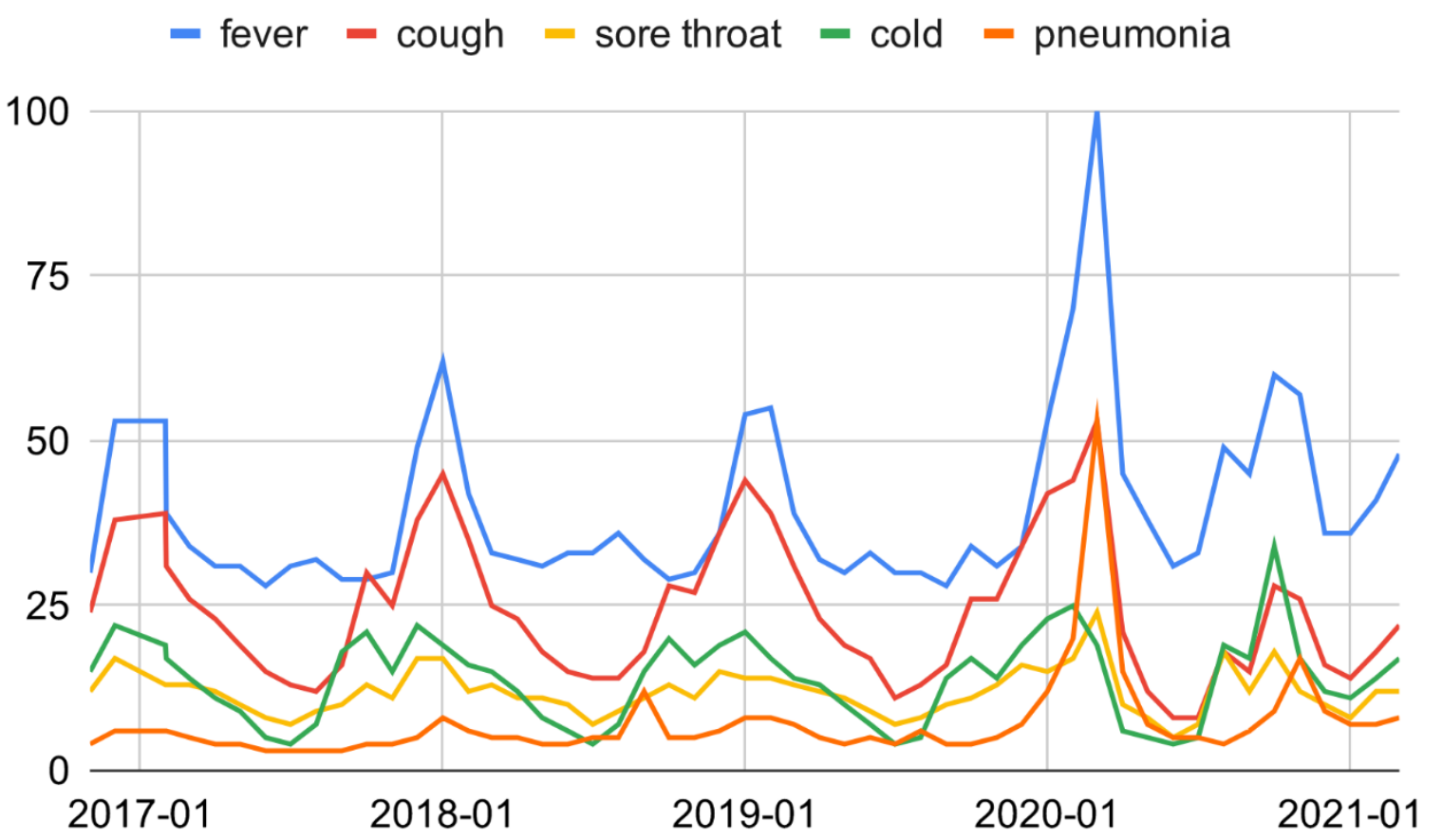

Supplementary Figure 1. Trend of COVID-19 symptoms-related web queries from 2017 to 2021 (Google Trends). The relative search volumes shown are cumulative monthly. 